\title{
Vortex Stabilization of Market Equilibrium in Theory and in Practice of Economics
}

\author{
A. JAKIMOWICZ ${ }^{a}$ AND J. JUZWISZYN ${ }^{b}$ \\ ${ }^{a}$ Institute of Economics, Polish Academy of Sciences, \\ Palace of Culture and Science, 1 Defilad Sq., PL-00-901 Warsaw, Poland \\ ${ }^{b}$ Department of Mathematics and Cybernetics, Wroclaw University of Economics \\ Komandorska 118/120, PL-53-345 Wrocław, Poland
}

\begin{abstract}
Rotary movements of the object around the position of equilibrium is the most common type of dynamics in nature. The way of plotting trajectory resembles winding a line onto a cone of revolution or some other solid of revolution. The state of equilibrium, which is usually not reached by the system, is marked with the cone axis. The trajectory can move away from the state of equilibrium, or get closer to it. A similar behavior is observed in many two-dimensional economic models, both linear, and nonlinear. The simplest example is a linear cobweb model, where - depending on slopes of linear demand function and linear function of supply - price and quantity make a broken line with a growing, constant or decreasing amplitude around the equilibrium point. In nonlinear models, trajectories are more realistic. A natural space for exploring spiral trajectories is a three-dimensional space. Usually, it requires magnifying the model's dimension by one. Economic vortices are made up by economic vectors of three constituents. It may be price, quantity, and time. Apparently, flat zigzags that can be seen on two-dimensional graphs of cobweb models are orthogonal projections of spinning trajectories. Vortexes created by nonlinear models are much smoother than the vortices created by linear models. The real economic vectors create smooth spiral trajectories, which indicates necessity to employ nonlinear dynamics in economic modeling. The basis for rotary movements are surface areas of solids of revolution of the second degree. The kinematics of solids indicated by market shows that they also rotate in three-dimensional space. It resembles precession movements. In economic dynamics we have at least a double rotation. What rotates are both economic vectors as well as the solids created by them.
\end{abstract}

PACS: 89.65.Gh, 45.40.-f, 47.32.C-

\section{Introduction}

The article presents theoretical and empirical exemplifications of the rotary movement of markets. The cobweb model and the Goodwin model of class struggle are a theoretical justification for this type of economic dynamics [1-5]. The latter is based on the well-known Lotka-Volterra model in population biology [6-8]. Experience shows that markets and economy reach states of equilibrium very rarely. Analyzing economic vectors in three dimensions, where the coordinates are price, volume, and time, confirms that vortex motions take place around virtual states of equilibrium. The view on stability of equilibrium stems from a long tradition of describing phenomena and economic processes by means of linear models. It was Pereto who first become concerned with basing the theory of general equilibrium on the rule of linearity, hence apart from real motions that lead to equilibrium, he also pinpointed contrary virtual motions, which - even though, in his opinion, do not exist in reality - should be investigated in order to explain the properties of given economic states [9]. This antinome was later explored by Samuelson, who came up with a solution referred to as the correspondence principle [10]. In a short period of time, the spin of economic vectors in a three-dimensional space can be described in accordance with the rules of kinematics of a rigid body. In this case, the states of virtual equilibrium are the lines of a trend that change in time. In the long run, trajectories of the vortex motion become irregular; what is more, their direction of twist can change, which makes it very difficult to describe those phenomena mathematically. The irregular rotations of stock exchange vectors resemble rotation of a jelly-like body, such as gelatin. The lateral surface of such a solid looks like a sleeve, on which there are many fields of concavity and convexity. This type of rotational dynamics resembles slightly a turbulent flux of fluid. Thus, the description of the trajectory by means of cones of regular precession, determined by the axes of a rotating solid is only an approximation of a real dynamics of stock exchange valid for a short period of time. The considerations found here constitute a further elaboration of our earlier research [11-12].

\section{Cobweb model as a cognitive archetype of contemporary economics}

The cobweb model is one of basic economic dynamic models and it analyzes prices and quantity dynamics on a single market. It was almost simultaneously formulated by Ricci, Schultz, and Tinbergen [13-15]. The name "cobweb" was introduced by Kaldor, because that is the shape taken by the graphic representation of prices and quantity adjustment process [16]. The first systematic elaboration of this theory is provided by Ezekiel [17]. The 
assumption here is that consumers react to a given event exactly at the moment of its manifestation on a market. Producers on the other hand, react to market changes after some time, i.e. with some delay. The cobweb model helps explain adjustment processes given a delayed reaction of producers to the changes in prices. The fluctuations result from interaction between the function of demand dependent on current prices and the function of supply dependent on prices expected by producers. In this model entrepreneurs do not remember anything but experiences from the previous period. According to a classic assumption adjusting the supply to a new price requires passing of some pre-fixed period, which can lead to one of three types of prices oscillation and volume of production: of stable amplitude, explosive and dampened. A market system is stable only in the last case. Manning develops this approach by introducing conditions of stability, permanent fluctuations, and instability for nonlinear functions of supply and demand [18].

The linear cobweb model is very often analyzed in economic literature [19-21]. The following two equations explain how the market works:

$$
\begin{aligned}
& q_{t}^{d}=D\left(p_{t}\right), \\
& q_{t}^{s}=S\left(p_{t-1}\right), \\
& q_{t}^{d}=q_{t}^{s},
\end{aligned}
$$

where the quantities $q_{t}^{d}$ and $q_{t}^{s}$ stand for demand and supply respectively, for a given good in period $t$, whereas $p_{t}$ is the prices of this period. Equation (1) features a classic dependence between demand and supply: demand is the function of current prices. According to equation (2) there is a one-period delay on the supply's side. The reason for it is the fact that production of many goods is not a momentary process, but it requires some period, which constitutes a unit of time measurement in the model. Producers decide on the size of production in the current period, based on the prices from a previous period. Relation (3) reveals a market balance in every period $t$. Usually the demand is a decreasing and supply an increasing function of prices. If both functions cross, it is possible to determine the price of balance $p_{e}$. If we consider the relations (1)-(3) and assume that there is a reverse function to the function of demand, the dynamics of prices is described by the first-order differential equation:

$$
p_{t}=D^{-1}\left[S\left(p_{t-1}\right)\right] .
$$

The phenomenon of cobweb is featured graphically in Fig. 1. Time paths of prices are orthogonal projections of vortex trajectories. In this simple model, we have three types of price fluctuations: the fading oscillations, with constant amplitude and explosive. In any situation, the equilibrium price $p_{e}$ is a reference system. Types of fluctuations depend on the slopes of demand and supply functions, expressed in absolute values. Since in the supply and demand functions (1)-(2) price is an independent variable, slopes are measured in relation to the $p$ axis. Let us consider in more detail the case of

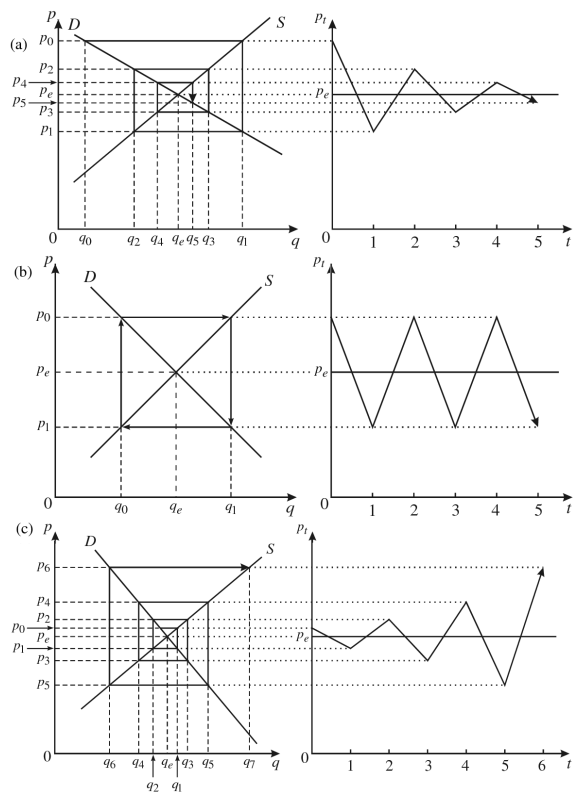

Fig. 1. Graphic representation of linear cobweb model: (a) dampened oscillations, (b) constant amplitude oscillations, (c) explosive oscillations.

dampened oscillations in prices, which are shown in Figure 1(a). The slope of demand function is larger than the slope of supply function. In the initial period $t_{0}$ there occurred some exogenous disturbance, such as crop failure, which knocked the system out of equilibrium position. As a result, the starting price $p_{0}$ is higher from the equilibrium price $p_{e} . q_{0}$ means the starting quantity. The price $p_{0}$ will make entrepreneurs manufacture produce of quantity $q_{1}$, which will constitute the supply of period 1. In order for the balance condition to be met, the demand will also equal $q_{1}$, which is true for the price $p_{1}$. This price sets up the supply in period 2 at a $q_{2}$ level. Such quantity will be absorbed when demand equals $q_{2}$, however, it requires price $p_{2}$. With price $p_{2}$ we have the supply at $q_{3}$ in period 3 . It will match the demand with the price at $p_{3}$, which will set supply for the next period at the $q_{4}$ amount. Repeating this reasoning allows us to determine prices and quantities in subsequent periods. Over time, the difference between $p_{t}$ and $p_{e}$ decreases, thus the time line of prices is convergent with the level of equilibrium. The adaptation process takes place as a result of emergence and disappearance of many moderately flexible regular short-term supply curves [22]. The intersection point of supply and demand curves $E_{0}$ temporarily changes its location. The process of spinning of positions of temporary equilibrium points lasts as long as it is subject to short-time stability $\left(p_{n}, q_{n}\right)=E_{n} \approx E_{0}$. However, in practice, rotation of vector $x=\left(p_{i}, q_{i}, t_{i}\right)$ is a constant process, since there is always some noise in the system which disturbs equilibrium.

The cobweb model is the archetype of modern cognitive theory of economics because it is a useful starting 
point for more advanced applications [23, 24]. Initially, a natural field of its application was economics of agriculture, but eventually its usefulness was discovered in modeling such phenomena as economic fluctuations or financial markets $[25,26]$. Therefore, we think that the product (vector) $x$ is a stock-exchange product (stock market shares or stock market index). Prices and volumes of thus understood vector $x$ are subject to very rapid changes.

\section{Market equilibrium stability in the Walrasian model}

In order to simplify the analysis process of a flat spin vector $x$, let us assume that demand $d\left(p_{t}\right)$ and supply $s\left(p_{t}\right)$ functions are still linear functions, where the variable is the price $p_{t}$ :

$$
\begin{aligned}
& d\left(p_{t}\right)=a_{d} p_{t}+b_{d}, \\
& s\left(p_{t}\right)=a_{s} p_{t}+b_{s}, \\
& \frac{\mathrm{d} p}{\mathrm{~d} t}=p^{\prime}(t)=c_{w}\left[d\left(p_{t}\right)-s\left(p_{t}\right)\right] .
\end{aligned}
$$

In addition let us assume that: $a_{d}<0, a_{s}>0, b_{d}>b_{s}$ and $c_{w}>0$. Equation (7) is referred to as the price postulate of Walras. This is a non-homogeneous differential equation with constant coefficients. Parameter $c_{w}$ can be called a parameter of rotational dynamics of the market. A large value of this parameter indicates rapid changes in the dynamics of price and volume of good $x$ [27].

According to Walras a market is in economic equilibrium if supply and demand are even. This seemingly static definition in fact is based on an assumption of a dynamic character, since it postulates the existence of economic forces, which affect such variables as price and quantity. According to the Walrasian postulate increases or decreases of price are expressed by the following inequalities:

$$
\begin{aligned}
& \frac{\mathrm{d} p}{\mathrm{~d} t}=p^{\prime}(t)>0 \Leftrightarrow d\left(p_{t}\right)>s\left(p_{t}\right), \\
& \frac{\mathrm{d} p}{\mathrm{~d} t}=p^{\prime}(t)<0 \Leftrightarrow d\left(p_{t}\right)<s\left(p_{t}\right), \\
& \frac{\mathrm{d} p}{\mathrm{~d} t}=p^{\prime}(t)=0=p_{e} \Leftrightarrow d\left(p_{t}\right)=s\left(p_{t}\right) .
\end{aligned}
$$

The market is in a state of equilibrium if the price of equilibrium $p_{e}$ meets the dependency $d\left(p_{e}\right)=s\left(p_{e}\right)$, thus $a_{d} p_{e}+b_{d}=a_{s} p_{e}+b_{s}$, hence $p_{e}=\frac{b_{s}-b_{d}}{a_{d}-a_{s}}$.

Price function in the Walrasian model is as follows:

$$
p(t)=\left(p_{0}-p_{e}\right) \exp \left[c_{w}\left(a_{d}-a_{s}\right) t\right]+p_{e},
$$

whereas $p(0)=p_{0}$. If $p_{0}=p_{e}$, then function $p(t)=$ $p_{e}=$ const. If, on the other hand, the directional coefficients of linear function of demand and supply satisfy these inequalities $a_{d}<0, a_{s}>0$ and $c_{w}>0$, then $c_{w}\left(a_{d}-a_{s}\right)<0$. Consequently, we get

$$
\lim _{t \rightarrow \infty} \exp \left[c_{w}\left(a_{d}-a_{s}\right) t\right]=0,
$$

which gives us

$$
\begin{aligned}
& \lim _{t \rightarrow \infty} p(t)=\left(p_{0}-p_{e}\right) \lim _{t \rightarrow \infty} \exp \left[c_{w}\left(a_{d}-a_{s}\right) t\right]+p_{e} \\
& \quad=p_{e} .
\end{aligned}
$$

The boundary value of price function explains market adaptation to the level of the temporary equilibrium [28]. In the model of a balanced market $\left(d\left(p_{e}\right)=s\left(p_{e}\right)\right)$ the above boundary relation has the following economic interpretation. If the price $p_{e}$ deviated in a certain period from the designated level of equilibrium $p_{0} \neq p_{e}$, then as a result of market operation, it will automatically seek that level. Function of prices in the Walrasian model (11) is a heterogeneous solution of differential equations with constant coefficients [29]:

$$
\frac{\mathrm{d} p}{\mathrm{~d} t}=p^{\prime}(t)=c_{w}\left(a_{d}-a_{s}\right) p(t)+c_{w}\left(b_{d}-b_{s}\right)
$$

with conditions

$$
\begin{aligned}
& a=c_{w}\left(a_{d}-a_{s}\right)<0, \\
& b=c_{w}\left(b_{d}-b_{s}\right)>0, \\
& p_{e}=\frac{-b}{a} .
\end{aligned}
$$

Inhomogeneous differential equation of price function reads:

$$
\frac{\mathrm{d} p}{\mathrm{~d} t}=p^{\prime}(t)=a p(t)+b .
$$

Its solution is shown in the following sequence of equalities:

$$
\begin{aligned}
p(t) & =\int b \exp \left(-\int a \mathrm{~d} t\right) \mathrm{d} t \exp \left(\int a \mathrm{~d} t\right) \\
+c & \exp \left(\int a \mathrm{~d} t\right), \\
p(t) & =b \int \exp (-a t) \mathrm{d} t \exp (a t)+c \exp (a t), \\
p(t) & =\frac{-b}{a} \exp (-a t) \exp (a t)+c \exp (a t), \\
p(t) & =\frac{-b}{a}+c \exp (a t), \\
p\left(t_{0}\right) & =p_{0}, \quad p_{0}=c \exp \left(a t_{0}\right)-\frac{b}{a}, \\
p(t) & =\left(p_{0}+\frac{b}{a}\right) \exp a\left(t-t_{0}\right)-\frac{b}{a} .
\end{aligned}
$$

If $t_{0}=0$ and $P(0)=P_{0}$ then the solution of equation (24) reads:

$$
p(t)=\left(p_{0}+\frac{b_{d}-b_{s}}{a_{d}-a_{s}}\right) \exp c_{w}\left(a_{d}-a_{s}\right) t-\frac{b_{d}-b_{s}}{a_{d}-a_{s}}
$$

\section{Rotary nature of markets}

Solution of homogenous differential equation of price

$$
\frac{\mathrm{d} p}{\mathrm{~d} t}=p^{\prime}(t)-a p(t)=0
$$

reads $p(t)=p_{0} \exp (a t)$. It is easy to note at this point the similarity between the solution of equation (26) and the equation of the curve called the logarithmic spiral, which is marked in polar coordinates $(r, \phi)$ and it is defined by the equation below 


$$
r(\phi)=a \exp (\lambda \phi)
$$

where: $a, \lambda>0$, whereas $\lambda=\operatorname{ctg} \psi$, however $\psi-$ is a constant angle formed by any ray emerging from a pole of the spiral with the tangent. If one considers the conditions (15) - (16), equation of price function takes the form of equation (11). Inequalities (15) - (16) are satisfied in the vortex-spiral model. This means that regardless of how big the difference between $p_{0}$ and $p_{e}$ is, the boundary equality is always satisfied $\lim _{t \rightarrow \infty} p(t)=p_{e}$. Price knocked out of equilibrium $p_{e}$ spins back in time to the equilibrium level.

Let us assume that there are two different functions $u(t)$ and $v(t)$, which solve the differential equation $\frac{\mathrm{d} p}{\mathrm{~d} t}=$ $p^{\prime}(t)=a p(t)+b$ with initial conditions

$$
\begin{aligned}
& u(0)=u_{0}, \quad v(0)=v_{0}: \\
& u(t)=\left(u_{0}-p_{e}\right) \exp \left[c_{w}\left(a_{d}-a_{s}\right) t\right]+p_{e}, \\
& v(t)=\left(v_{0}-p_{e}\right) \exp \left[c_{w}\left(a_{d}-a_{s}\right) t\right]+p_{e} .
\end{aligned}
$$

The boundary of the absolute difference between these functions is equal to

$$
\lim _{t \rightarrow \infty}\left|u_{0}-v_{0}\right| \exp \left[c_{w}\left(a_{d}-a_{s}\right) t\right]=0 .
$$

Thus, any two solutions over time get closer to each other - their difference in the value of $t$ decreases to zero. True is also a monotonous (poor) estimate of this difference

$$
\begin{aligned}
& |u(t)-v(t)|=\left|u_{0}-v_{0}\right| \exp \left[c_{w}\left(a_{d}-a_{s}\right) t\right] \leq\left|u_{0}-v_{0}\right|, \\
& \quad \forall t \geq 0,
\end{aligned}
$$

which means that the difference in the function value $u\left(t_{0}\right)$ and $v\left(t_{0}\right)$ at the initial point determines the size of their difference along the entire positive time-axis . In addition one can notice that if $a=c_{w}\left(a_{d}-a_{s}\right)>$ 0 (it is the so-called turbulent market case), then for $p_{e} \neq p_{0}$ a finite boundary $\lim _{p \rightarrow \infty} p(t)$ does not exist. Then even a minor difference between $u_{0}$ and $v_{0}$ will cause a significant rise in the absolute value $|u(t)-v(t)|$, if $t \rightarrow \infty$. In this situation, the market will reveal a very large "turbulent" dynamics of the vector $x$ prices [30]

\section{Regular precession as a short-term approximation of market dynamics}

Fig. 2 features a modified cobweb model. If we look at the sets of flat trajectory of vortex systems in the long term, it is easy to associate that their shapes are orthogonal projections of helices wound on a variety of regular precession cones, which are formed by freely rotating rigid bodies.

If a rigid body moves in such a way that the momentary motion is always combined of two rotations around two intersecting axes, the first of which $l$ is fixed in space, while the other $m$ has a fixed position in the body, wherein the angular velocity $\boldsymbol{\omega}_{1}, \boldsymbol{\omega}_{2}$ of these rotations is constant in magnitude, such a rigid body motion is called regular precession (Fig. 3). Since by assumption axes $l$ and $m$ intersect at point $e$, so the momentary motion

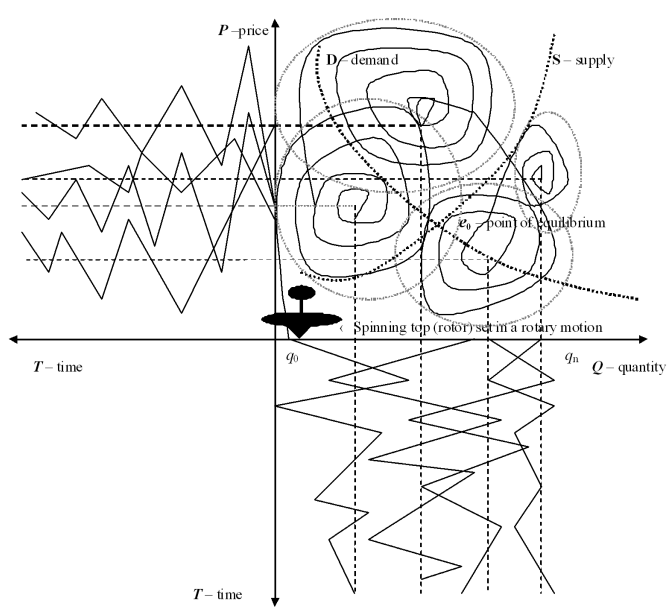

Fig. 2. Spiral-vortex trajectories designated by the kinematics of vector $x$ in a modified cobweb model.

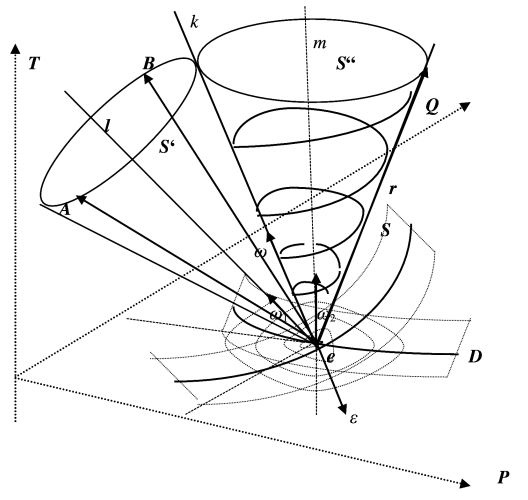

Fig. 3. Regular precession. Rotations of economic vector $\boldsymbol{x}=\left(p_{i}, q_{i}, t_{i}\right)$, with components: price, quantity, time, around the economic equilibrium line marked and crossing through point $e$ in $\mathrm{R}_{+}^{3}=\boldsymbol{P} \times \boldsymbol{Q} \times \boldsymbol{T}$. Real model of vortex equilibrium of vector $\mathrm{x}$.

of a rigid body is a rotation with an angular velocity $\boldsymbol{\omega}=\boldsymbol{\omega}_{1}+\boldsymbol{\omega}_{2}$ around the $k$ axis, which passes through the point of intersection of the axes $l$ and $m$. Note that the momentary motion of the axis $m$ is a momentary rotation around the axis $l$ with angular velocity $\boldsymbol{\omega}_{1}$ (rotation of the axis $m$ around itself is out of the question.) Thus, the axis $m$ rotates around the axis $l$ with constant angular velocity $\boldsymbol{\omega}_{1}$. The intersection point $e$ of both axes is thus fixed and the angle between the axes $l$ and $m$ is constant. As a result vector $\boldsymbol{\omega}$, and so axis of momentary rotation $k$ is at constant angles with the axes $l$ and $m$. Axis $k$ makes a rotating cone $S^{\prime}$ in space. The trace of $k$ axis in the body is also a rotation cone $S^{\prime \prime}$. Thus, cones of temporary rotations axis, constant and movable, are cones of rotation with axes $l$ and $m$.

Vector of angular acceleration $\boldsymbol{\varepsilon}=\boldsymbol{\omega}_{1} \times \boldsymbol{\omega}$ can be determined taking into consideration the fact that $\boldsymbol{\omega}=\boldsymbol{\omega}_{1}+\boldsymbol{\omega}_{2}$ we will easily conclude that $\boldsymbol{\varepsilon}=\boldsymbol{\omega}_{1} \times \boldsymbol{\omega}_{2}$ ( since $\boldsymbol{\omega}_{1} \times \boldsymbol{\omega}_{1}=$ $0)$. The vector of angular acceleration $\varepsilon$ is of course per- 
pendicular to angular velocity vectors: $\boldsymbol{\omega}_{1}, \boldsymbol{\omega}_{2}$. Knowing the momentary position of rotation axis and components of angular velocity of the rigid body around this axis, we can determine linear velocity vector of any point on the surface of a rigid body [31]. It is clear that in short periods of time the rotating real-exchange vectors determine the cones at $\mathrm{R}_{+}^{3}=\boldsymbol{P} \times \boldsymbol{Q} \times \boldsymbol{T}$. Consequently, let us consider the vector $\boldsymbol{x}=\left(p_{i}, q_{i}, t_{i}\right)$ as a rotating solid:

$$
\boldsymbol{V}=\boldsymbol{\omega} \times \boldsymbol{r}=\left|\begin{array}{ccc}
\boldsymbol{i} & \boldsymbol{j} & \boldsymbol{k} \\
\omega_{p} & \omega_{q} & \omega_{t} \\
p & q & t
\end{array}\right|
$$

It is known that the vector of linear acceleration $\boldsymbol{a}=$ $\boldsymbol{a}_{1}+\boldsymbol{a}_{2}$ equals the geometric sum of precession acceleration vector $\boldsymbol{a}_{1}=\boldsymbol{\varepsilon} \times \boldsymbol{r}=\left(\boldsymbol{\omega}_{1}+\boldsymbol{\omega}_{2}\right) \times \boldsymbol{r}$ and axis acceleration vector $\boldsymbol{a}_{2}=\boldsymbol{\varepsilon} \times \boldsymbol{V}=\left(\boldsymbol{\omega}_{1}+\boldsymbol{\omega}_{2}\right) \times \boldsymbol{V}$. Based on the above, let us imagine that a circular cone with apex angle $2 \alpha=\frac{\pi}{3}$ and the length of the forming side wall equal to $h$ is rolling without slipping over a horizontal plane $0 P Q$. The axis of the cone rotates at a constant angular velocity of precession around the vertical axis $0 T$. It is possible to calculate both speed and linear acceleration of points $A$ and $B$ for such a moving a cone on a plane. Suppose further that the tip of the ongoing free cone, located at a point $0 P Q T$. The lengths of the vectors $0 A$ and $0 B$ have the following values $\boldsymbol{r}_{A}=\frac{1}{2} h(\boldsymbol{j}+\boldsymbol{k} \sqrt{3}), \boldsymbol{r}_{B}=\frac{1}{2} h\left(\boldsymbol{i}+\frac{2}{3} \boldsymbol{j}+\frac{1}{2} \boldsymbol{k} \sqrt{3}\right)$ respectively, and the values of angular velocity vectors are equal to $\boldsymbol{\omega}_{1}=\omega_{1} \boldsymbol{k}, \boldsymbol{\omega}=-\omega_{1} \boldsymbol{j} \sqrt{3}$. Thus the movement velocity of vector $A$ can be written as follows:

$$
\begin{gathered}
\boldsymbol{V}_{A}=\boldsymbol{\omega} \times \boldsymbol{r}_{A}=\left|\begin{array}{ccc}
\boldsymbol{i} & \boldsymbol{j} & \boldsymbol{k} \\
0 & -1 & 0 \\
0 & 1 & \sqrt{3}
\end{array}\right| \omega_{1} \sqrt{3} \frac{h}{2}=-\frac{3}{2} \omega_{1} h \boldsymbol{i}, \\
\boldsymbol{V}_{A}=-\frac{3}{2} \omega_{1} h .
\end{gathered}
$$

Similarly, one can calculate the motion velocity of vector $V_{B}$ :

$$
\begin{gathered}
\boldsymbol{V}_{B}=\boldsymbol{\omega} \times \boldsymbol{r}_{B}=\left|\begin{array}{ccc}
\boldsymbol{i} & \boldsymbol{j} & \boldsymbol{k} \\
0 & -1 & 0 \\
1 & \frac{3}{2} & \frac{1}{2} \sqrt{3}
\end{array}\right| \omega_{1} \sqrt{3} \frac{h}{2} \\
=\omega_{1} h\left(-\frac{3}{4} \boldsymbol{i}+\frac{1}{2} \boldsymbol{k} \sqrt{3}\right), \quad V_{B}=\frac{1}{4} \omega_{1} h \sqrt{21} .
\end{gathered}
$$

The value of the angular acceleration vector of the cone $\varepsilon$ is:

$$
\begin{gathered}
\boldsymbol{\varepsilon}=\boldsymbol{\omega}_{1} \times \boldsymbol{\omega}=\left|\begin{array}{ccc}
\boldsymbol{i} & \boldsymbol{j} & \boldsymbol{k} \\
0 & 0 & 1 \\
0 & -1 & 0
\end{array}\right| \omega_{1}^{2} \sqrt{3}=\omega_{1}^{2} \boldsymbol{i} \sqrt{3} \\
\boldsymbol{\varepsilon}=\omega_{1}^{2} \sqrt{3}
\end{gathered}
$$

The values of linear acceleration vectors of points $A$ and $B$ are respectively:

$$
\begin{aligned}
& \boldsymbol{a}_{1 A}=\boldsymbol{\varepsilon} \times \boldsymbol{r}_{A}=\left|\begin{array}{ccc}
\boldsymbol{i} & \boldsymbol{j} & \boldsymbol{k} \\
0 & -1 & 0 \\
0 & 1 & \sqrt{3}
\end{array}\right| \omega_{1}^{2} \sqrt{3} \frac{h}{2} \\
& =\frac{1}{2} \omega_{1}^{2} h(-3 \boldsymbol{j}+\boldsymbol{k} \sqrt{3}), \\
& \boldsymbol{a}_{2 A}=\boldsymbol{\omega} \times \boldsymbol{V}_{A}=\left|\begin{array}{ccc}
\boldsymbol{i} & \boldsymbol{j} & \boldsymbol{k} \\
0 & -1 & 0 \\
-1 & 0 & 0
\end{array}\right| \omega_{1}^{2} \sqrt{3} \frac{3 h}{2} \\
& =-\frac{3}{2} \omega_{1}^{2} h\left(\boldsymbol{i}+\boldsymbol{k} \frac{\sqrt{3}}{2}\right), \\
& \boldsymbol{a}_{A}=\boldsymbol{a}_{1 A}+\boldsymbol{a}_{2 A}=-\omega_{1}^{2} l\left(\frac{3}{2} \boldsymbol{j}+\boldsymbol{k} \sqrt{3}\right), \\
& \boldsymbol{a}_{1 B}=\boldsymbol{\varepsilon} \times \boldsymbol{r}_{B}=\left|\begin{array}{ccc}
\boldsymbol{i} & \boldsymbol{j} & \boldsymbol{k} \\
1 & 0 & 0 \\
1 & \frac{3}{2} & \frac{\sqrt{3}}{2}
\end{array}\right| \omega_{1}^{2} \sqrt{3} \frac{h}{2} \\
& =\frac{3}{4} \omega_{1}^{2} h(-\boldsymbol{j}+\boldsymbol{k} \sqrt{3}), \\
& \boldsymbol{a}_{2 B}=\boldsymbol{\varepsilon} \times \boldsymbol{V}_{B}=\left|\begin{array}{ccc}
\boldsymbol{i} & \boldsymbol{j} & \boldsymbol{k} \\
0 & -1 & 0 \\
\frac{-3}{4} & 0 & \frac{\sqrt{3}}{2}
\end{array}\right| \omega_{1}^{2} h \sqrt{3} \\
& =\frac{-3}{2} \omega_{1}^{2} h\left(\boldsymbol{i}+\boldsymbol{k} \frac{\sqrt{3}}{2}\right), \\
& \boldsymbol{a}_{B}=\boldsymbol{a}_{1 B}+\boldsymbol{a}_{2 B}=-\omega_{1}^{2} l\left(\frac{3}{2} \boldsymbol{i}+\boldsymbol{j}_{\frac{3}{4}}\right) .
\end{aligned}
$$

\section{Market vortices in practice and their theoretical description in economics}

Flat vortices can be seen in those charts, that feature correlations between the values of stock indices and their corresponding values of the volumes. Spiral trajectories of this type are shown in Figure 4. Charts volumes and index values are plotted on two time scales: yearly for the DJIA and monthly for the NIKKEI 225. In these periods indices made both a clockwise and anticlockwise spiral. Fig. 5 shows a vortex trajectory in $\mathrm{R}_{+}^{3}=\boldsymbol{P} \times \boldsymbol{Q} \times \boldsymbol{T}$ set by vector $x$ (of WIG20 index) of the following components $\left(p_{i}, q_{i}, t_{i}\right)$. At present a working hypothesis is being tested that vortex trajectories form a logarithmic spiral.

Such kind of dynamics has a binary justification in the theory of economics. It can be a result of trajectory rotation around the point of the Cournot-Nash equilibrium in the triopoly model, which takes place along the ever-tightening spiral [12]. The potential swelling and shrinking of the spiral can be attributed to exogenous and stochastic factors. Another way to theoretically describe this topic is to use the class struggle model by Goodwin [32-34]. In this sense, capitalism has always been in constant lap motion, which results from antagonistic relations of production. 

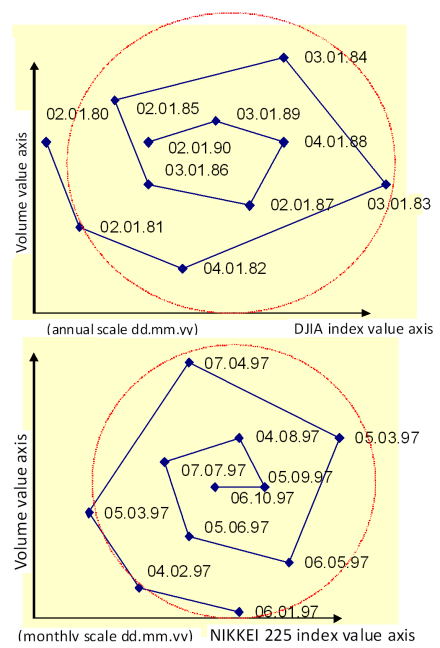

Fig. 4. Vortex trajectories of DJIA NYSE and NIKKEI 225 indexes.

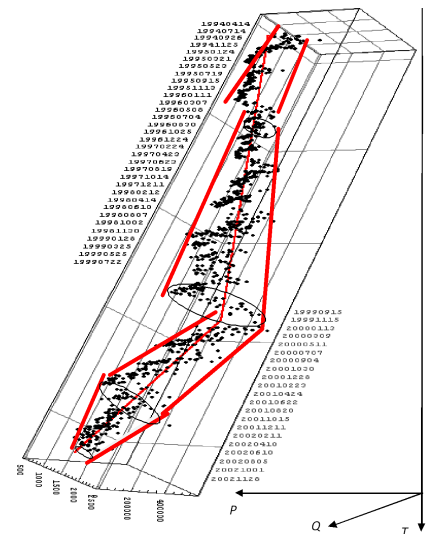

Fig. 5. Three-dimensional vortex trajectory of WIG20 index from 14.04.1994 to 28.11.2002, designating side surfaces of rotating bodies (cones).

\section{Conclusions}

The easy-to-calculate values of velocity, as well as the linear accelerations of any points $A$ and $B$ situated at the surface of the cone of a regular precession determined by the axis of rotation of a rotating solid can be used only when the vortex trajectory marks the cone at $R_{+}^{3}$, and such situations take place only in short periods of time. Then, the surfaces determined by the vortex trajectory are close to the surface of cones. In the long periods of time, the rotating vectors determine very irregular vortex trajectories, which are of various directions of twist, which gravely complicates any attempt at describing the vortex motion, alongside of which the $x$ vector is going to move in the future. The chaotic and long-lasting character of vortex trajectories shows the kind of motion that can be named an irregular topological precession, of course an irregular precession of a non-solid. As an example for such a body one can imagine a rotating piece of freezing in time gelatin. The lateral surface of a solid, which results from a chaotic and supposedly smooth rotation of a "gelatin-like" $x$ vector, has a shape of an irregular "sleeve" of stock exchange and it is full of various areas of concavity and convexity. The complexity of the above-presented dynamics of chaotic rotation of the $x$ vector seems to be more complicated than the problem of stability of our Solar System, whose solution Poincaré dared to find [35]. Exploring the complexity of motion he understood that he had made a serious mistake in his paper on the celestial mechanics that was awarded by the king of Sweden and Norway, Oscar II. The mistake was soon to be corrected. Therefore, the notion of chaos was better understood and the very process of its better description lasts until now.

\section{References}

[1] R.G.D. Allen, Ekonomia matematyczna, PWN, Warszawa 1961.

[2] G. Gandolfo, Mathematical Methods and Models in Economic Dynamics, North-Holland, Amsterdam 1971.

[3] R.M. Goodwin, in: Ed. C.H. Feinstein, Socialism, Capitalism and Economic Growth. Essays Presented to Maurice Dobb, Cambridge University Press, Cambridge, 54 (1967).

[4] R. Veneziani, S. Mohun, Structural Change and Economic Dynamics 17, 437 (2006).

[5] G. Colacchio, M. Sparro, C. Tebaldi, International Journal of Bifurcation and Chaos 17, 1911 (2007).

[6] A.J. Lotka, Proc. Natl. Acad. Sci. USA 6, 410 (1920).

[7] A.J. Lotka, Proc. Natl. Acad. Sci. USA 7, 168 (1921).

[8] V. Volterra, Leçons sur la théorie mathématique de la lutte pour la vie, Gauthier-Villars, Paris 1931.

[9] V. Pareto, Manual of Political Economy, Macmillan, London 1971.

[10] P.A. Samuelson, Foundations of Economic Analysis (Enlarged edition), Harvard University Press, Cambridge, Mass. 1983.

[11] J. Juzwiszyn, Wiry ekonomiczne $i$ fale R.N. Elliotta, Katedra Matematyki i Cybernetyki, Wydział Zarządzania i Informatyki, Akademia Ekonomiczna im. Oskara Langego, Wrocław 2006.

[12] A. Jakimowicz, Źródła niestabilności struktur rynkowych, Wydawnictwo Naukowe PWN, seria: Współczesna Ekonomia, Warszawa 2010.

[13] U. Ricci, Zeitschrift für Nationalökonomie, Band I, Heft 5, 649 (1930).

[14] H. Schultz, Der Sinn der statistischen Nachfragekurven, in: Ed. E. Altschul, Veröffentlichungen der Frankfurter Gesellschaft für Konjunkturforschung, Heft 10, Kurt Schroeder Verlag, Bonn 1930.

[15] J. Tinbergen, Zeitschrift für Nationalökonomie, Band I, Heft 5, 669 (1930).

[16] N. Kaldor, The Review of Economic Studies 1, 122 (1934).

[17] M. Ezekiel, The Quarterly Journal of Economics 52, 255 (1938). 
[18] R. Manning, The Economic Record 46, 588 (1970).

[19] G. Gandolfo, Economic Dynamics: Methods and Models, North-Holland, Amsterdam 1980.

[20] W.D. Dechert, Ed., Chaos Theory in Economics: Methods, Models and Evidence, Edward Elgar Publishing, Cheltenham 1996.

[21] A. C. Chiang, Podstawy ekonomii matematycznej, PWE, Warszawa 1994.

[22] G. Akerman, The Quarterly Journal of Economics 71, 151 (1957).

[23] C.H. Hommes, Chaotic Dynamics in Economic Models. Some Simple Case-Studies, Wolters-Noordhoff, Groningen 1991.

[24] C.H. Hommes, Journal of Economic Behavior and Organization 24, 315 (1994).

[25] P.E. Smith, International Economic Review 8, 1 (1967).

[26] M.G. Allingham, Economica 43, 169 (1976).

[27] J. Juzwiszyn, Ekonomia Matematyczna 5, 133 (2001).

[28] J. Juzwiszyn, W. Rybicki, Niektóre modele matematyki ubezpieczeniowej $i$ finansowej. Statystyka $i$ ryzyko. Praktyka statystyki, Wydawnictwo Akademii Ekonomicznej im. Oskara Langego we Wrocławiu, Wrocław 2006.
[29] T. Żylicz, Wykłady z równań różniczkowych i różnicowych, Wydawnictwo Uniwersytetu Warszawskiego, Warszawa 1986.

[30] J. Tadion, Rozszyfrować rynek, WIG Press, Warszawa 1999.

[31] S. Banach, Mechanika, Spółdzielnia Wydawnicza Czytelnik, Warszawa 1947.

[32] N.H. Barbosa-Filho, L. Taylor, Metroeconomica 57, 389 (2006).

[33] N.J. Moura, M.B. Ribeiro, Eur. Phys. J. B 67, 101 (2009).

[34] M.M. García, M.E. Herrera, Cuadernos de Economía 29, 1 (2010).

[35] H. Poincaré, Sur le problème des trois corps et les équations de la dynamique, in: Oeuvres de Henri Poincaré, Vol. VII: Masses fluides en rotation. Principes de mécanique analytique. Problème des trois corps, Gauthier-Villars, Paris 262 (1952). 